\title{
Evaluating the impacts of autonomous cars on the capacity of freeways in Brazil using the HCM-6 PCE methodology
}

\section{Avaliação dos impactos de veículos autônomos na capacidade de rodovias no Brasil pelo método de equivalência veicular do HCM-6}

\author{
Renan Favero', José Reynaldo Setti ${ }^{2}$ \\ ${ }^{1}$ University of São Paulo, São Carlos, São Paulo - Brazil, renan.favero@alumni.usp.br \\ ²University of São Paulo, São Carlos, São Paulo - Brazil, jrasetti@usp.br
}

\section{Recebido:}

13 de agosto de 2020

Aceito para publicação:

22 de abril de 2021

Publicado:

24 de agosto de 2021

Editor de área:

Flávio Cunto

\section{Keywords:}

Autonomous vehicles.

Capacity.

HCM-6.

CAF.

Simulation.

Passenger-car equivalent.

\section{Palavras-chave:}

Veículos autônomos.

Capacidade.

HCM-6.

CAF.

Simulação.

Fator de equivalência veicular.

DOI:10.14295/transportes.v2912.2444

OPEN

ACCESS

\begin{abstract}
This paper analyses the factors that affect the impact of autonomous vehicles (AVs) on the capacity of a freeway in Brazil using an adaptation of the HCM-6 procedure for truck PCE estimation. A version of Vissim, recalibrated to represent traffic streams and AVs on Brazilian freeways, was used to simulate more than 25,000 scenarios representing combinations of traffic (e.g., AV fleets, AV platoons, percentage of AVs and of heavy goods vehicles) and road (grades and number of lanes) characteristics. AV impacts on capacity were evaluated by means of the capacity adjustment factor (CAF) and a model to estimate CAF from control variables was fitted and validated. The results indicate increases of up to $30 \%$ in capacity with $60 \%$ of platooning-capable AVs. Statistical analyses show that the fraction of $A V s$ in the stream and the proportion of platooning-capable AVs are the factors with the greatest impact on this increase in capacity.
\end{abstract}

\section{RESUMO}

Este artigo analisa os fatores que afetam os impactos dos veículos autônomos (AVs) na capacidade de uma autoestrada no Brasil adaptando o método usado no HCM-6 para obter equivalentes veiculares de caminhões. Uma versão do Vissim, recalibrada para representar veículos convencionais e AVs em rodovias brasileiras, foi usada para simular mais de 25.000 cenários, representando combinações de características do tráfego (frotas de AVs, pelotões de AVs, porcentagem de AVs e de caminhões) e da via (rampas e número de faixas). $O$ impacto dos $A V s$ foi avaliado através do coeficiente de ajuste da capacidade (CAF) e um modelo para estimar o valor do CAF com base nas variáveis de controle foi ajustado e validado. Os resultados sugerem acréscimos na capacidade de até $30 \%$, com $60 \%$ de AVs capazes de formar pelotões. Análises estatísticas mostram que os fatores mais significativos neste aumento de capacidade são a fração de AVs no tráfego e a proporção de AVs capazes de formar pelotões.

\section{INTRODUCTION}

One of the attractions of autonomous vehicles (AVs) is the increase in capacity due to the more intensive use of traffic lanes, and consequent improvements in the efficiency of road operations (Zhao and Sun, 2013; Bierstedt et al., 2014). Various studies suggest that AVs will be able to improve traffic flow with a high level of penetration in traffic streams and cooperation 
(VanderWerf and Miller, 2004; Shladover et al., 2012; Shladover, 2009). However, the effects of AVs on the capacity of freeways with a low and medium level of penetration are not yet well known (Milanes et al., 2014).

Understanding the impact of AVs in a wide range of motorway scenarios is of the utmost importance for concessionaires and public agencies responsible for motorway system operations. How to incorporate AVs when assessing the capacity and quality of service is an aspect that should be considered by managers, mainly for freeways that are under concession contracts and where the maintenance of a minimum quality standard is guaranteed under contract. In Brazil, as in many other countries, the quality of services is assessed by the Highway Capacity Manual (HCM), which still does not provide an instrument that includes AVs in the process, in its most recent edition, HCM-6 (TRB, 2016).

The present study is part of a broader project, which proposes a way to include AVs in quality-of-service assessments based on the HCM- 6 passenger-car equivalents (PCE) method. This paper discusses the effects that different aspects of freeways, traffic and AVs have on capacity. The impacts considered include: the type of AVs and their penetration rate; the maximum number of platooning vehicles; the number of lanes; the proportion of heavy goods vehicles (HGV) in the traffic stream; and the length and magnitude of the slopes. In this study, a microsimulation software was used to generate synthetic vehicle flow data. Based on the capacity of each scenario, the capacity adjustment factor (CAF) and a model for estimating the $C A F$ value as a function of the simulation control variables were obtained.

\section{THEORETICAL FRAMEWORK AND PROPOSED APPROACH}

While the concept of connected and autonomous vehicles is broad, in this study, the classification proposed by the CoExist project (Sukennik, 2018) will be used. CoExist defines AVs as driverless automobiles, which are classified as: cautious, normal and all-knowing, depending on their driving behaviour. The cautious AV type behaves more cautiously than human-driven vehicles (HDV). The normal AV type behaves similarly to HDVs. All-knowing vehicles are platooning-capable AVs that have better awareness and predictive capabilities of manoeuvres and more cooperative behaviour (Sukennik, 2018).

The main impacts expected from AVs in the traffic stream include: an increase in the maximum flow, fewer lane changes, more homogeneous and stable flow, and a reduction of traffic jams (Calvert et al., 2017). AVs can travel with smaller headways and with variable spacing depending on the kind of vehicle travelling in front of them (Shi et al., 2019; Makridis et al. 2018; Martin-Gasullaet al., 2019). When connected, the AVs exchange information (such as: distance, speed, acceleration and failure events), allowing them to be safer and respond faster (Van Arem et al., 2006). All these characteristics result in an increase in road capacity (Mahmassani, 2016; Zhao and Sun, 2013); the size of this increment, however, differs in each study as it depends on the road's characteristics and the behavioural parameters used in the simulations since there is not yet a sufficient number of these vehicles in traffic (Zhao and Sun, 2013, Shi et al., 2019). Therefore, simulations that take into consideration the local traffic characteristics are essential for more accurate analyses.

The reduction in lane change manoeuvres and the deterministic behaviour of AVs result in less conflicts and more homogeneous traffic (Strand et al. 2011, Gorter, 2015; Papadoulis, 2019; Alkim et al., 2007). Therefore, the presence of AVs increases the space mean speed of the traffic stream and reduces traffic jams (Makridis et al., 2018). Although the different impacts of AVs 
have already been studied by and large, there is a lack of studies in the literature that address the combined effects of road characteristics, AV fleet composition and AV platooning capabilities.

As AVs are still in the testing phase, it is expected that there will be a long period of coexistence between HDVs and AVs in low proportions and at different levels of automation on freeways (Mahmassani, 2016; Calvert et al., 2017; Shi et al., 2019). Thus, this research focuses on analysing the impacts on freeway capacity at levels of low and medium penetration of AVs in the traffic stream.

This article reports on a broader study, whose aim is to propose incorporating AVs in the assessment of the quality of services on freeways and expressways in Brazil, based on the HCM6 methodology for PCE estimation. In the proposed approach, the effect of AVs is treated in a similar way to that used to incorporate the effect of HGV on the quality of services: using an equivalence factor. In the case of HGV, HCM-6 adjusts the demand by (TRB, 2016, p. 12-33):

$$
v_{p}=\frac{V}{P H F \cdot N \cdot f_{H V}},
$$

where $v_{p}$ is the equivalent demand flow rate $(\mathrm{pc} / \mathrm{h} / \mathrm{ln}) ; V$ is the observed hourly volume (veh/h); $P H F$ is the peak hour factor; $N$ is the number of lanes; and $f_{H V}$ is the adjustment factor for the HGV effect, which is calculated by (TRB, 2016, p. 12-34):

$$
f_{H V}=\frac{1}{1+P_{T}\left(E_{T}-1\right)}
$$

where $P_{T}$ is a fraction of HGV in the traffic and $E_{T}$ an equivalence factor.

On HCM-6, the vehicle equivalent $E_{T}$ is calculated following a complex process which basically consists of (Zhou et al., 2019a; List et al., 2015): (1) obtaining flow-density graphs for base traffic (automobiles only) and mixed traffic (cars+HGV); (2) obtaining the capacity adjustment factor ( $C A F$ ); (3) Adjusting the $C A F$ prediction model; (4) estimating the $C A F$ values for specific conditions; and (5) estimating the $E_{T}$ values for specific conditions of the road and traffic. As the method calculates equivalents for the same capacity (List et al., 2015), HCM-6 defines the capacity adjustment factor $\left(C A F_{\text {mix }}\right)$ as the $C A F_{\text {mix }}=C_{\text {mix }} / C_{\text {base }}$ ratio between $C_{\text {mix }}$, the maximum mixed flow (automobiles+HGV) and $C_{\text {base }}$, the maximum flow of cars. In the case of HGV, the value of $C A F_{\text {mix }}$ is always lower than 1 and is used to obtain the equivalence factor of $\mathrm{HGV}$ $E_{T}$ through (Zhou et al., 2019b):

$$
E_{T}=\frac{1-\left(1-P_{T}\right) C A F_{\text {mix }}}{P_{T} \cdot C A F_{\text {mix }}} .
$$

The authors propose adapting the HCM-6 method using an adjustment factor $\left(f_{A V}\right)$ for the presence of AVs in traffic, which would be calculated using:

$$
f_{A V}=\frac{1}{1+P_{A V}\left(E_{A V}-1\right)}
$$

where $P_{A V}$ is the fraction of AVs in the flow, and $E_{A V}$ is an equivalence factor for the AVs. Therefore, the adjustment of the demand value is done by including an extra factor into Eq. 1:

$$
v_{p}=\frac{V}{P H F \cdot N \cdot f_{A V} \cdot f_{H V}}
$$

where the adjustment factor for the presence of AVs, $f_{A V}$, represents the effect of a particular set of traffic stream (percentage of heavy vehicles, percentage of AVs and the composition of the AVs fleet) and the road (number of lanes, magnitude and slope length, etc.) conditions in the same way that $f_{H V}$ represents the effect of HGV for the specific conditions of the analysed stretch: fraction of HGV and magnitude and slope length (TRB, 2016; p. 12-34). 
This paper analyses the factors that affect the $C A F$ on major freeways in Brazil and proposes a model for its prediction (stages 1 to 3 of the HCM- 6 method); the estimate of the equivalence factor values $E_{A V}$ for highways and expressways in Brazil will be addressed in another paper.

\section{METHOD}

This research is a prospective study, which uses synthetic data generated by simulations, as AVs are still in the testing phase. Briefly, the adopted method can be described by the following steps: (1) obtaining the recalibrated microsimulation software to represent traffic flow on Brazilian freeways; (2) defining the experiment and simulation scenarios; (3) generating synthetic data through simulations; (4) calculating the $C A F$ for each scenario; (4) analysing the effect of controlled variables on CAF; (5) adjusting the CAF prediction model; and (6) validating the prediction model.

The synthetic data were obtained from simulations carried out in Vissim 2020, which is able to simulate AVs (Sukennik, 2018), where behavioural and vehicle performance parameters of automobiles and HGV were recalibrated to better represent the traffic on Brazilian freeways (Bethônico et al, 2016; Carvalho and Setti, 2019). The following sections present the research steps.

\section{GENERATING SYNTHETIC DATA}

This section describes how synthetic traffic flow data were obtained using the Vissim software.

\subsection{Simulation Model}

The network used (Table 1) consists of three segments. The first one is $4 \mathrm{~km} \mathrm{long}$, it is flat, and allows vehicles to reach the segment under study in a stable way. The data are collected in an intermediate link which is $8 \mathrm{~km}$ long, where the slope and number of lanes vary according to the scenario. Five sensors, installed at 500, 1000, 2000, 4000 and $8000 \mathrm{~m}$ from the beginning of the link, are used to collect the data. Positioned in this way, the sensors can simulate five different lengths of the gradient without needing to change the total length of the road in each simulation. The exit segment is $2 \mathrm{~km}$ long and has a slope of $-2 \%$.

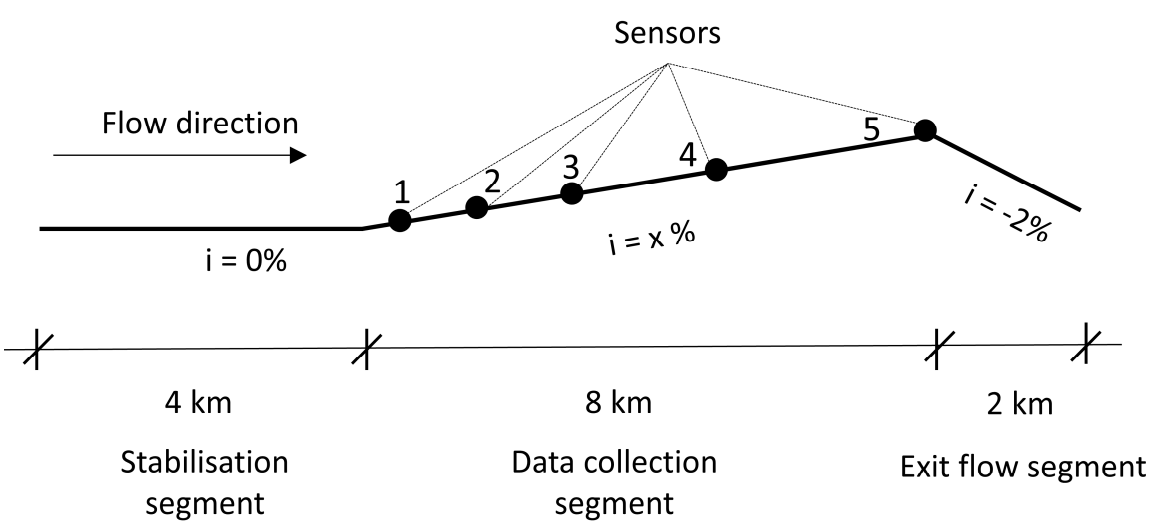

Figure 1. Representation of the simulated segment (without scale).

The time-step of the simulations was adopted as 5 updates/second to obtain the best combination between the most realistic results and computational performance (PTV, 2019). 
Conventional automobiles, i.e., human-driven vehicles (HDVs) were simulated using parameters from the Wiedemann-99 model, previously calibrated for typical highways in the state of São Paulo (Bethônico et al., 2016). Recalibrated parameters are those related to the carfollowing (CF) and lane-changing (LC) models and to the desired speed. The Table 1 provides parameter values for the CF and LC models used in this study and compares them to the Vissim default values. The "HDVs" column contains the recalibrated values (Bethônico et al., 2016) and the "cautious", "normal" and "all-knowing" columns contain the recalibrated values in the CoExist project (Sukennik, 2018).

Table 1 - Parameter values of the Vissim car-following (CF) and lane-changing (LC) models, adopted for this study

\begin{tabular}{rrrrrrr}
\hline Parameter & Description of Parameter (Model) & Default & HDVs & Cautious & Normal & All-knowing \\
\hline OBSVEH & Observed Vehicles (CF) & 2,00 & 4,00 & 1,00 & 1,00 & 8,00 \\
CCO & Standstill distance (CF) & 1,50 & 2,56 & 1,50 & 1,50 & 1,00 \\
CC1 & Following distance (CF) & 0,90 & 0,75 & 1,50 & 0,90 & 0,60 \\
CC2 & Longitudinal oscillation (CF) & 4,00 & 7,13 & 0,00 & 0,00 & 0,00 \\
CC3 & Perception threshold for following (CF) & $-8,00$ & $-12,92$ & $-10,00$ & $-8,00$ & $-6,00$ \\
CC4 & Positive speed difference (CF) & $-0,35$ & $-0,54$ & $-0,10$ & $-0,10$ & $-0,10$ \\
CC5 & Negative speed difference (CF) & 0,35 & 0,69 & 0,10 & 0,10 & 0,10 \\
MH & Min. headway - front/rear (LC) & 0,50 & 1,75 & 0,00 & 0,00 & 0,00 \\
SD & Safety distance reduction factor (LC) & 0,60 & 0,39 & 0,10 & 0,10 & 0,10 \\
MD & Max. decel. for cooperative braking (LC) & $-3,00$ & $-1,28$ & $-3,00$ & $-3,50$ & $-4,00$ \\
TSL & To slower lane if collision time above (LC) & 0,00 & 3,11 & 1,20 & 1,50 & 2,00 \\
\hline
\end{tabular}

The desired speed distribution for HDVs was recalibrated to better represent Brazilian drivers' behaviour based on data collected from a one-hour video of a freeway, recorded in the off-peak period. The space mean speeds were obtained using a method based on computer vision (Marcomini and Cunha, 2018). Only passenger cars travelling under flow rates below 500 veh/h/ln (TRB, 2016; p. 12-27) and headways higher than 8 seconds in relation to the vehicle in front (Al-Kaisy and Durbin, 2011) were included in the sample, making a total of 205 cars. The desired speed distribution for HDVs, shown in Figure 2, was obtained from the cumulative frequency observed for this sample. The distribution obtained indicates that the studied stream has vehicles that are slower than those in the distribution adopted as default by Vissim.

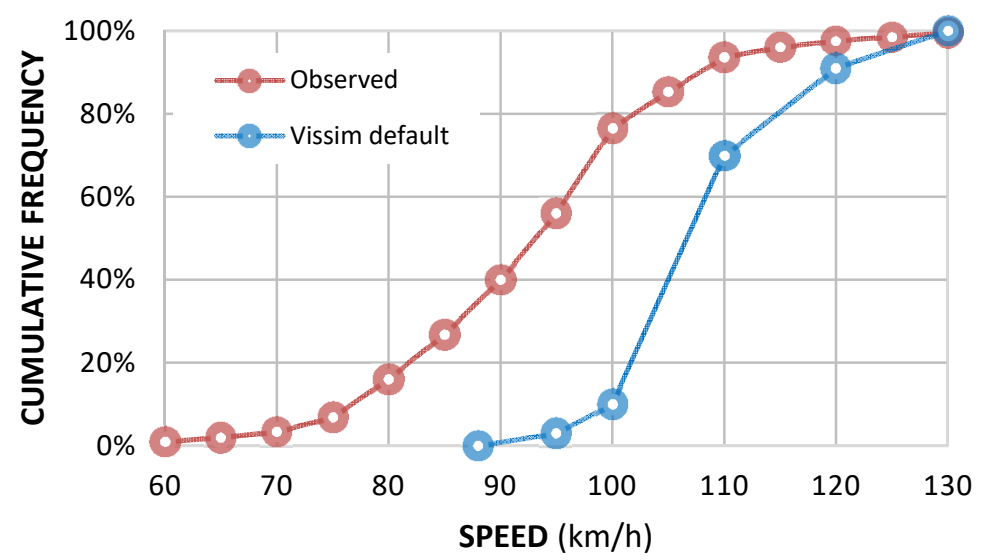

Figure 2. Desired speed distributions for conventional vehicles (HDVs), N=205. 
The recalibrated parameters for HGV performance were obtained from a previous study (Carvalho and Setti, 2019) and include: maximum and desired acceleration functions, cumulative weight and power functions, and dimensions. These parameters were input into the software for four categories of HGV that characterise fleets of HGV that travel on freeways in São Paulo state (Carvalho and Setti, 2019).

Simulated AVs are automobiles of three types: cautious, normal and all-knowing $-A V_{1}, A V_{2}$ and $A V_{3}$, respectively. Each type of $\mathrm{AV}$ represents a different behaviour and $\mathrm{V} 2 \mathrm{~V}$ communication level; therefore, they have different calibration parameters, as well as distinct observation and cooperation capabilities (Table 1). For example, while $A V_{1}$ and $A V_{2}$ are able to see only the vehicle in front of them and do not form platoons, $A V_{3}$ monitors up to 8 vehicles ahead and presents cooperative behaviour during lane changes and has the ability to form platoons (Sukennik, 2018). The default values of Vissim were used for the parameters related to the formation of $\mathrm{AV}$ platoons: maximum distance of $250 \mathrm{~m}$ to start a platoon; and 0.6 second headways and a minimum distance of $2 \mathrm{~m}$ from the platoon. $A V_{1}$ vehicles have the enforce absolute braking distance option activated (Sukennik, 2018).

All AVs were simulated using deterministic, rather than a stochastic operation. The desired speed for AVs was defined as the speed limit of $\pm 2 \mathrm{~km} / \mathrm{h}$. The headway of the $A V_{3}$ depends on the vehicle in front. In order to maintain smaller headways, these vehicles have an increase in the acceleration factor, defined as $105 \%$ for the $A V_{2}$ and $110 \%$ for the $A V_{3}$. These characteristics make the $\mathrm{AV}_{3}$ vehicle an $\mathrm{AV}$ with cooperative behaviour. This configuration for the three types of AVs enables us to evaluate the effect of the behaviour of AVs on capacity and, consequently, on the equivalence factor.

\subsection{Simulated scenarios}

In order to evaluate the impact of AVs on different traffic conditions, the experiment considers a large number of scenarios combining controlled variables, shown in Table 2.

The Autonomous variable represents the total percentage of AVs in the flow, whose maximum value was set at $60 \%$, as AVs are not expected to reach levels close to $100 \%$ of the flow in the near future (Calvert et al., 2017). Traffic streams containing only HDVs are base flow for a given scenario and traffic streams containing HDVs and AVs are mixed flows for that scenario. The AV fleet could comprise different proportions of AV types. In this experiment, 9 compositions were established with different proportions for each type of $\mathrm{AV}$, as shown in Table 3, in such a way that the sum of proportions of $A V_{1}+A V_{2}+A V_{3}$ always totalled $100 \%$.

Table 2 - Controlled variables of the experiment

\begin{tabular}{llll}
\hline Controlled variable & Abbreviation & Levels & Level description \\
\hline Percentage of AVs in the stream & Autonomous & 7 & $0 \%, 10 \%, 20 \%, 30 \%, 40 \%, 50 \%, 60 \%$ \\
Proportion of type 1 AVs (cautious) & AV1 & 4 & $0 \%, 25 \%, 50 \%, 75 \%\left({ }^{1}\right)$ \\
Proportion of type 2 AVs (normal) & AV2 & 4 & $0 \%, 25 \%, 50 \%, 75 \%\left({ }^{1}\right)$ \\
Proportion of type 3 AVs (all-knowing) & AV3 & 4 & $0 \%, 25 \%, 50 \%, 75 \%\left({ }^{1}\right)$ \\
Distance travelled on the slope (m) & Distance & 5 & $500,1000,2000,4000,8000$ \\
Number of lanes & Lanes & 3 & $2,3,4$ \\
Maximum number of AVs in the platoon & Platoon & 4 & $0,2,4,8$ \\
Longitudinal slope & Ramp & 3 & $0 \%, 2 \%, 4 \%$ \\
Proportion of HGV in the flow & HGV & 3 & $15 \%, 30 \%, 45 \%$ \\
\hline (') Percentages &
\end{tabular}

(') Percentages such as AV1 + AV2 + AV3 $=100 \%$, as shown in Table 3 
The characteristics related to the road were also controlled in the simulation scenarios. The scenarios were created using different numbers of lanes on the freeway segment $(2,3$ and 4 ), as well as the gradient magnitude, which can be $0 \%,+2 \%$ and $+4 \%$. These variables are relevant in this study as they affect the quality of service on freeways with a high number of heavy vehicles, as is the case in Brazil (Carvalho and Setti, 2018). Moreover, the literature does not show studies that address the effect of slopes on traffic streams with AVs.

The combinations of control variables to provide a total number of 5103 scenarios, of which: 567 were scenarios without AV platoons (first 3 AV combinations in Table 3, 7 levels of proportion of AVs in the stream, 3 levels of traffic lanes, 3 levels of ramps, and 3 levels of percentage of heavy vehicles) and 4536 were scenarios with AV platoons (6 remaining AV combinations, 7 levels of proportion of AVs, 4 levels of platoon size, 3 levels of traffic lanes, 3 levels of ramps, and 3 levels of percentage of heavy vehicles). The time chosen for the simulation was 90 minutes: 30 minutes for warming-up and the remaining time for data collection. Each scenario was simulated 5 times, with approximately 50\%,60\%, 75\%, 90\% and 100\% of the maximum possible flow in the stream, which was previously set.

Table 3 - Combinations of AV types used in the experiment

\begin{tabular}{llllllllll}
\hline \multirow{2}{*}{ AV type } & \multicolumn{2}{c}{ Combination (AV fleet composition) } \\
\cline { 2 - 9 } & 1 & 2 & 3 & 4 & 5 & 6 & 7 & 8 & 9 \\
\hline $\boldsymbol{A} \boldsymbol{V}_{\mathbf{1}}$ (cautious) & $75 \%$ & $50 \%$ & $25 \%$ & $25 \%$ & $25 \%$ & $25 \%$ & $0 \%$ & $0 \%$ & $0 \%$ \\
$\boldsymbol{A} \boldsymbol{V}_{\mathbf{2}}$ (normal) & $25 \%$ & $50 \%$ & $75 \%$ & $50 \%$ & $25 \%$ & $0 \%$ & $75 \%$ & $50 \%$ & $25 \%$ \\
$\boldsymbol{A} \boldsymbol{V}_{\mathbf{3}}$ (all knowing) & $0 \%$ & $0 \%$ & $0 \%$ & $25 \%$ & $50 \%$ & $75 \%$ & $25 \%$ & $50 \%$ & $75 \%$ \\
\hline
\end{tabular}

Considering that the large number of scenarios to be simulated and the method used for calculating the $E_{T}$ values used in HCM does not foresee replications of the simulations to generate synthetic data (Zhou et al., 2019b), it was initially decided to evaluate the need for replications of the simulations. As empirical results indicate that the number of replications required depends on the variable analysed and that, in some cases, one or two replications are sufficient (Fries et al. 2014), 6 scenarios were simulated with 9 replications (different random seeds). The scenarios that were chosen randomly for this analysis correspond to the following conditions: $30 \%$ of heavy vehicles; level road; two traffic lanes; $50 \%$ of AVs, in three different compositions 4, 5 and 6 (Table 3) and capable of forming platoons of up to two AVs; and four distances travelled in the link (500, 1000, 2000 and $4000 \mathrm{~m})$.

For each of the replications, the $C A F$ values and their averages were calculated. Then, those values were normalised (Table 4) in such a way that the average would become equal to 1 . Considering this, the variation of the values obtained for $C A F$ can be evaluated in relation to the average for all 54 cases. By observing the last line of Table 4, it can be seen that the greatest variation observed between the normalised average (1.00) and the $C A F$ normalised values was $3.6 \%$. This variation margin can be considered small for an exploratory study such as this one. Thus, considering the computational costs involved in the replication of the simulations and the time available for this research, it was decided to reproduce the procedure used for calculating the equivalence factors of HCM (Zhou et al., 2019b), where no replications were made.

In total, the generation of synthetic data required 25,515 simulations, which were carried out automatically using a program written in Python that prepared the input files for the Vissim simulation and collected the results from each simulation. 
Table $4-C A F$ normalised values obtained from simulations of 6 scenarios with 9 replications

\begin{tabular}{|c|c|c|c|c|c|c|}
\hline \multirow{3}{*}{ Replication } & \multicolumn{6}{|c|}{ Combination (AV fleet composition) } \\
\hline & 4 & 5 & 6 & 4 & 5 & 6 \\
\hline & \multicolumn{3}{|c|}{ Distance $500 \mathrm{~m}$} & \multicolumn{3}{|c|}{ Distance $1000 \mathrm{~m}$} \\
\hline 1 & 1.018 & 0.994 & 1.003 & 0.997 & 1.000 & 1.021 \\
\hline 2 & 1.033 & 1.022 & 1.017 & 1.036 & 0.999 & 0.993 \\
\hline 3 & 0.977 & 0.967 & 0.990 & 1.012 & 1.015 & 0.996 \\
\hline 4 & 1.019 & 1.008 & 1.004 & 1.011 & 0.987 & 1.007 \\
\hline 5 & 0.977 & 0.980 & 1.001 & 0.984 & 1.000 & 1.007 \\
\hline 6 & 1.005 & 1.022 & 1.004 & 1.012 & 1.001 & 0.996 \\
\hline 7 & 0.977 & 0.981 & 1.016 & 0.997 & 1.000 & 0.981 \\
\hline 8 & 0.990 & 1.006 & 0.988 & 0.970 & 0.999 & 1.006 \\
\hline 9 & 1.004 & 1.020 & 0.977 & 0.983 & 0.999 & 0.993 \\
\hline Average & 1.000 & 1.000 & 1.000 & 1.000 & 1.000 & 1.000 \\
\hline Max. variation & 0.033 & 0.033 & 0.023 & 0.036 & 0.015 & 0.021 \\
\hline
\end{tabular}

\section{FACTORS THAT AFFECT THE CAPACITY ADJUSTMENT FACTOR}

The $C A F$ was calculated for all simulated traffic streams. The capacity was defined as the flow rate of the $95^{\text {th }}$ percentile of the flow of each scenario, which was obtained from time intervals of 5 minutes of observation, as stipulated in HCM-6 (Dowling et al., 2014). Figure 3(a) shows an example, where one scenario without AV results in a capacity of $1692 \mathrm{veh} / \mathrm{h} / \mathrm{ln}$; Figure 3(b), shows that the capacity increases to $1878 \mathrm{veh} / \mathrm{h} / \mathrm{ln}$ when the stream has $60 \%$ of AVs.

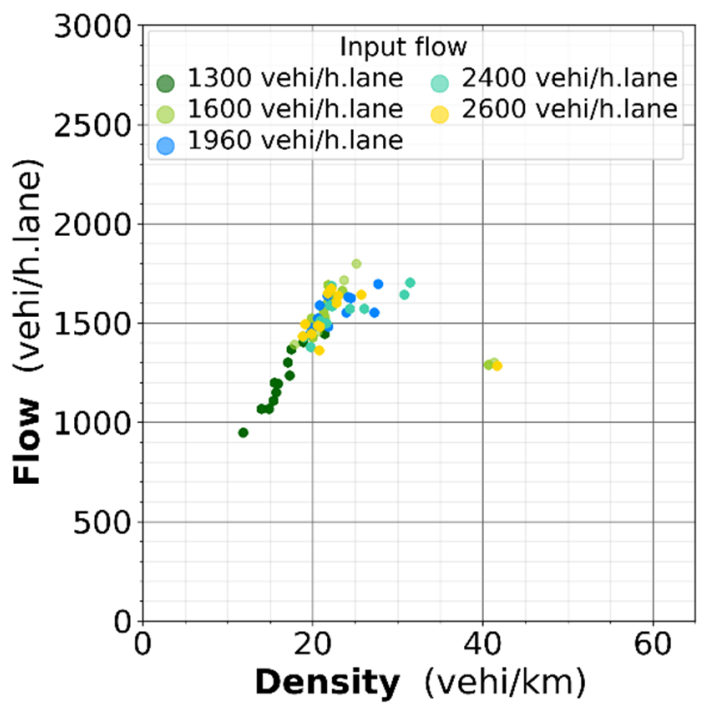

(a) $0 \% \mathrm{AVs}$ in the stream

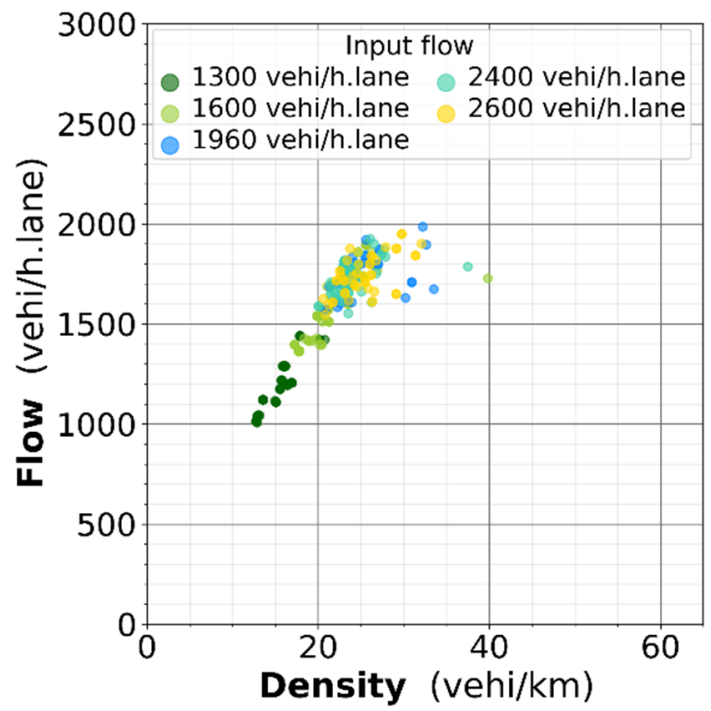

(b) $60 \% \mathrm{AVs}\left(25 \% A V_{2}\right.$ and $\left.75 \% A V_{3}\right)$

Figure 3. Capacity determination through the 95 th percentile of the flow-density ratio, for scenarios with $0 \%$ slope, $30 \% \mathrm{HGV}, 2$ lanes, a distance of $500 \mathrm{~m}$.

In this section the factors that affect the $C A F$ are analysed. The $C A F$ is the ratio between the capacity of the mixed flow (conventional vehicles and AVs) and the capacity of the base flow (only conventional vehicles):

$$
C A F_{i}=\frac{C_{i, \text { mix }}}{C_{i, \text { base }}}
$$

where $C A F_{i}$ is the $C A F$ for scenario $i ; C_{i, m i x}$ is the capacity (vehic/h) for the mixed flow scenario $i$; $C_{i, b a s e}$ is the capacity (vehic/h) for the base flow in scenario $i$. 


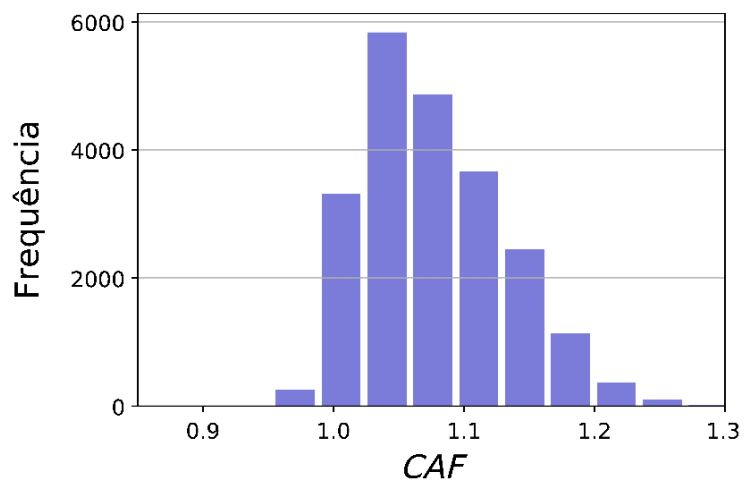

(a)

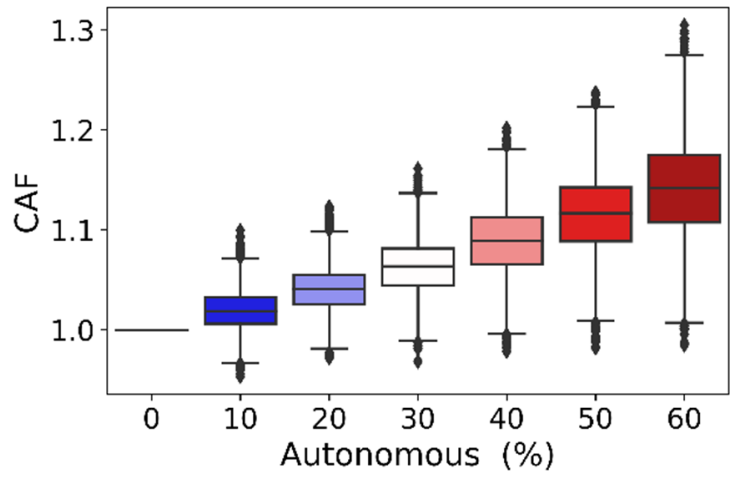

(b)

Figure 4. Characterisation of the $C A F(N=22005)$ values: (a) histogram of the CAF values observed and (b) boxplot showing the variation of the $C A F$ value as a function of the AV fraction in the traffic stream.

The histogram of the values found for $C A F$, shown in Figure 4(a), presents an asymmetric distribution whose average is 1.077 and the variance is $0.003(N=22005$, as the sample excludes the repeated base scenarios). As expected, this asymmetry is due to the larger number of observations greater than 1 , since in most simulated scenarios there was an increase in the capacity, given that the AVs are able to maintain smaller headways than those of conventional vehicles. The boxplot in Figure 4(b) indicates an increase in the variance as the proportion of AVs in the stream increases.

The simulated scenarios resulted in $C A F$ values between 0.95 and 1.30 . The maximum value represents a $30 \%$ increase in the capacity and was obtained in a scenario with a high proportion of $A V_{3}$, platooning-capable $A V s$. On the other hand, the lowest value was observed in scenarios with only $10 \%$ of non-platooning AVs. In these scenarios, the $C A F$ values were between 0.95 and 1.10. The CAF values, aggregated by the type of fleet and proportion of AVs in the flow (Figure 5 ), lead us to conclude that the higher the connectivity of the AVs, the higher the $C A F$ value (combination 9 has $75 \%$ of platooning-capable AVs); it can also be observed that the level of penetration of the AVs implies an average increase in the $C A F$ values.

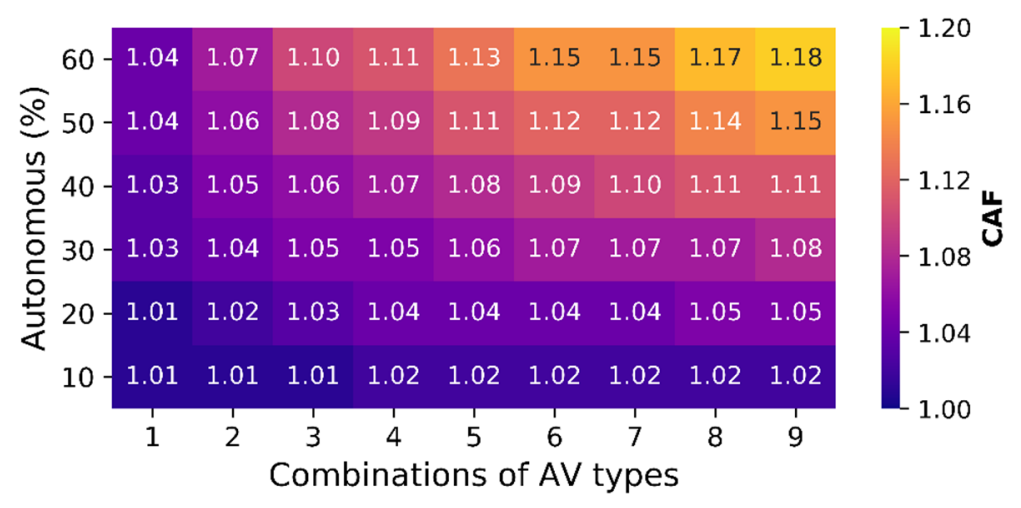

Figure 5. Heat map showing the variation of the CAF values observed as a function of the level of penetration of $A V s$ (\% Autonomous) and the combinations of AV types in the flow (shown in Table 3: Combinations of AV types used in the experiment. The values in the boxes are the average of the values in the lane.

The analysis of the effect of the parameters on the capacity was carried out using the $C A F$, which represents the impact of the AVs on the capacity. Welch's one-way ANOVA showed a major effect of the penetration of AVs (Autonomous) on the capacity: $F(6,21998)=54669.09$; 
$p<0.05$; and $\omega=0.77$. The boxplot in Figure(b) indicates that the impact of AVs on the capacity increases as the level of penetration increases. To test this hypothesis, as there is not variance homogeneity in the samples corresponding to each level of penetration $(0 \%, 10 \%, \ldots$, $60 \%$ ), the Games-Howel post-hoc test and Hochberg's GT2 test were used. The results show that the average of the $C A F$ values for each penetration level were significantly different from each other $(\alpha=5 \%)$, indicating that a $10 \%$ increase in the level of $\mathrm{AV}$ penetration contributes to an increase in capacity.

The factorial ANOVA was used to verify whether the control variables (Table 2) affected the $C A F$ values. The results (Table 5) indicate that for $\alpha=5 \%$, all control variables have a significant first order effect, except for the slope length - which can be explained as the performance of AVs is not affected by the slope. This table also shows that the second order effects were significant $(p<0.05)$, except for the second-order interaction Autonomous $\times$ Distance. The $\eta^{2}$ values show that the most important factors are the level of AV penetration and their capacity to form platoons.

Table 5 -Results of Factorial ANOVA, showing only the significant first and second order effects at the $5 \%$ confidence level

\begin{tabular}{lllll}
\hline Effect & G.L. & $\mathbf{F}$ & $\mathbf{p}$ & $\mathbf{\eta}^{\mathbf{2}}$ \\
\hline Autonomous & 6 & 7415.851 & $<0.001$ & 0.670 \\
Platoon & 3 & 1143.138 & $<0.001$ & 0.135 \\
Autonomous $\times$ HGV & 12 & 51.497 & $<0.001$ & 0.027 \\
Autonomous $\times$ Ramp & 12 & 32.613 & $<0.001$ & 0.018 \\
Autonomous $\times$ Lanes & 12 & 28.634 & $<0.001$ & 0.015 \\
HGV & 2 & 154.353 & $<0.001$ & 0.014 \\
Ramp & 2 & 83.050 & $<0.001$ & 0.008 \\
HGV $\times$ Lanes & 4 & 38.380 & $<0.001$ & 0.007 \\
Lanes & 2 & 49.358 & $<0.001$ & 0.004 \\
Autonomous $\times$ Distance & 24 & 0.988 & 0.501 & 0.001 \\
\hline $\mathrm{R}^{2}=0.707$ & & & & \\
$\mathrm{R}^{2}$ adjusted $=0.706$ & & & & \\
\hline
\end{tabular}

Interaction graphs were used to investigate the interaction between the main effects on the average $C A F$ value. The graphs in Figure 6 represent these interactions, comparing the results for the flows where there is predominance of platooning-capable AVs $\left(A V_{3}\right)$ with the flows where there is predominance of platooning-incapable AVs ( $A V_{1}$ and $\left.A V_{2}\right)$. Figure 6(a) shows that the $C A F$ rises as the fraction of AVs increases in both cases, although more markedly with the predominance of AV3 in the flow and that, in this case, there is a relation with the percentage of HGV.

An analysis of the comparisons between scenarios with different fleets, shown in Figure 6, shows that all the factors analysed have little influence on streams with a predominance of platooning-incapable AVs. In streams with a predominance of $A V_{3}$, these factors have a different behaviour. The graph in Figure 6(a) shows that increasing the percentage of HGVs reduces CAF; the graph in Figure 6(b) indicates that for scenarios with more than $30 \%$ of AVs, the more lanes there are, the smaller the increase in CAF, a phenomenon also observed in the literature (Xiaobao and Ning, 2007). Figure 6(c) suggests that steep gradients (4\% or more) affect CAF more than level segments (2\% or less), while the graph in Figure $6(d)$ indicates that the gradient length has no effect on CAF and the graph in Figure 6(e) shows that the size of the platoon has 
no impact on CAF, except for the scenario with a high rate of AVs. In scenarios with a predominance of $A V_{3}$ and $60 \%$ of AVs, scenarios with smaller maximum platoon length generated higher CAF values.

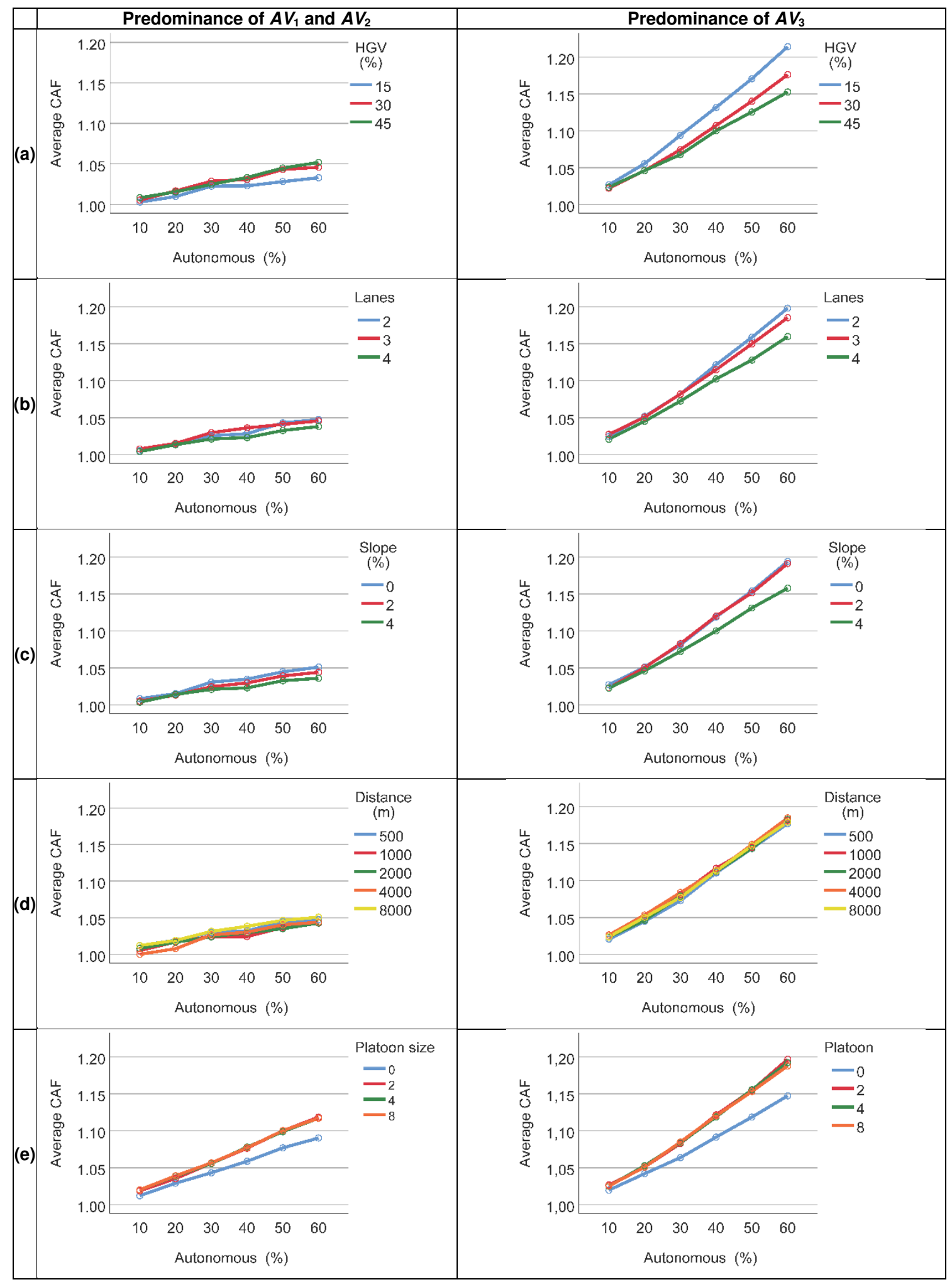

Figure 6. Combined effect of the composition of the fleet of AVs and of the control variables over the CAF: (a) percentage of HGV in the flow; (b)number of traffic lanes on the freeways; (c) magnitude of the ramp; (d) length of the slope; and (e) size of AV platoons. Different from AV3, AV1 and AV2 are non-platooning AVs 


\section{MODEL TO ESTIMATE VALUES FOR THE CAPACITY ADJUSTMENT FACTOR}

In HCM-6 (TRB, 2016, p. 26-6; Zhou, Rilett and Jones, 2019b), the CAF values are obtained using regression models. However, these models are complex, with parameters that are difficult to interpret and cannot be observed in a traffic stream. In order to overcome these limitations, we propose a model for estimating the $C A F$ which, can easily evaluate the effect of each parameter and covers all simulated scenarios.

\subsection{Calibration of the $C A F$ prediction model}

The model used to estimate the $C A F$ value was obtained by multiple linear regression, whose independent variables are listed in Table 2, except for the distance travelled on the slope, which does not influence the CAF, and the fraction of type 2 AVs (AV2). The AV2 variable was excluded from the model because it presents a high correlation with AV3 $(r=0.697)$ and a low correlation with CAF ( $r=0.029$ ), which is explained by the similarity of the behaviour parameters between $A V_{2}$ and HDVs.

The model obtained is statistically significant: $F(7 ; 21.997)=9077.57$ and $p<0.05$, with $R^{2}=0.743, R_{\text {adjusted }}^{2}=0.743$ and $r=0.862$. All coefficients are significant for $\alpha=5 \%$ and all parameters show a logical relation (Table 2). The adjusted model is:

$$
C A F=1.028+0.243 A V-0.065 A V_{1}+0.031 A V_{3}-0.064 P_{T}-0.322 R-0.005 F+0.001 P
$$

where $C A F$ : estimated value of the capacity adjustment factor; $A V$ : proportion of autonomous vehicles in the flow, in decimal values; $A V_{1}$ : proportion of cautious AVs in the flow, in decimal values; $A V_{3}$ : proportion of all-knowing AVs in the flow, in decimal values; $P_{T}$ : proportion of $\mathrm{HGV}$ in the flow, in decimal values; $R$ : slope of the ramp, in $\mathrm{m} / \mathrm{m} ; F$ : number of traffic lanes on the freeway, in the direction studied; and $P$ : maximum number of AVs in a platoon. Table 6 summarizes the model fitting results.

Table 6 - Results of the multiple linear regression, obtained using the stepwise method

\begin{tabular}{lllllll}
\hline Variables & $\mathbf{B}$ & $\boldsymbol{\beta}$ & $\mathbf{t}$ & $\mathbf{p}$ & Tolerance & VIF \\
\hline (Constant) & 1.000 & & 967.91 & $<0.01$ & - & - \\
AV & 0.243 & 0.77 & 225.50 & $<0.01$ & 1.00 & 1.00 \\
AV1 & -0.065 & -0.21 & -56.79 & $<0.01$ & 0.82 & 1.22 \\
PT & -0.064 & -0.15 & -42.47 & $<0.01$ & 1.00 & 1.00 \\
AV3 & 0.031 & 0.14 & 38.28 & $<0.01$ & 0.82 & 1.22 \\
R & -0.322 & -0.10 & -28.36 & $<0.01$ & 1.00 & 1.00 \\
F & -0.005 & -0.08 & -23.38 & $<0.01$ & 1.00 & 1.00 \\
P & 0.001 & 0.07 & 19.55 & $<0.01$ & 0.92 & 1.09 \\
\hline
\end{tabular}

The standardised coefficients (Table 6) show that the variable with the highest predictive power is $\mathrm{AV}$, which is compatible with the factorial ANOVA results. The $A V_{3}$ and $P$ variables, which represent the beneficial effect of the AV platoons on capacity, have positive coefficients. From the variables that have negative signs, which reduce the capacity, it should be noted that the increase of $A V_{1}$ (AVs with excessively conservative behaviour) in the traffic stream reduces the capacity, as well as the proportion of HGV. Figure 6(a)-(c) show the deleterious effect of increasing $R, P_{T}, F$ and $P$ in $C A F$. Table 6 also shows that the predictors do not present significant collinearity as the tolerance statistics were higher than 0.1 and the variance inflation factor (VIF) is less than 10. 


\subsection{Validation and analysis of the prediction model quality of the CAF}

The validation of the adjusted model was carried out with results from 2,340 scenarios which were simulated with random seeds different from the ones used in other simulations and three intermediate and different levels of penetration of AVs in the traffic stream. These changes were used to generate traffic streams different from those used for the model calibration. During the validation, the values for the other variables were not changed, as the experiment's objective was to evaluate the effect of AVs on the traffic flow. To obtain a smaller sample for validation, only three combinations of AVs were used (Table 3): combination 9, with a higher percentage of $A V_{3}$, platooning-capable AVs; combination 1, in which $A V_{1}$ predominates; and combination 4, a middle ground. Table 7 summarises the controlled variables used in the validation.

Table 7 - Controlled variables for model validation sample

\begin{tabular}{ll}
\hline Controlled variables & Adopted values \\
\hline Percentage of AVs & $0 \%, 15 \%, 35 \%, 55 \%$ \\
Compositions of AVs & 1,4 and 9 \\
Number of traffic lanes & 2,4 \\
Distance travelled on the slope $(\mathrm{m})$ & $500,1000,2000,4000,8000$ \\
Percentage of HGV & $15 \%, 30 \%, 45 \%$ \\
Maximum size of platoon & $0,2,4,8$ \\
Gradient magnitude & $0 \%, 2 \%, 4 \%$ \\
\hline
\end{tabular}

The validation was carried out comparing the $C A F$ values observed for the validation sample against the $C A F$ values estimated using the model in Equation 7. Figure 7(a) shows the relation between the observed and the estimated values (the band represents a tolerance margin of 4\%), whereas Figure 7(b) shows the proportion of correct answers in the model in Equation 7. Figure 7 (b) shows that $74 \%$ of the values predicted by the model were within the $4 \%$ error margin, compared to the observed values. Figure 7(c) and Figure 7(d) show that the model has a better ability to predict the $C A F$ value for scenarios with a higher proportion of type 3 AVs and produces larger errors in scenarios where type 1 AVs predominate.

The quality of the $C A F$ estimates was evaluated using the mean normalised error (MNE), mean absolute error (MAE), normalised root mean square error (NRMSE) and the correlation coefficient $(r)$.

The observed and estimated $C A F$ values presented a high correlation $(r=0.724)$ and a low value of systematic errors, $M N E=0.019$. As $M A E=0.032$ and $N R M S E=0.040$, indicating that the error in the $C A F$ prediction is $3 \%$ to $4 \%$, on average. Additionally, the standard deviation of the observed values $\sigma_{o b s}=0.048$ is very close to the standard deviation of the estimated values, $\sigma_{\text {est }}=0.051$, which indicates that the dispersions of the two sets are similar. The low error values demonstrate a good accuracy of the model, within the limits of the simulated values for the independent variables.

\section{FINAL CONSIDERATIONS}

The aim of the research reported here was to analyse the impact of AVs on the capacity of major freeways in Brazil, using a recalibrated version of Vissim to represent typical automobiles and HGV typical of Brazilian freeways. The capacity was obtained using the HCM-6 method and a wide range of control variables was used. The ANOVA results indicated that the larger the 
fraction of AVs in traffic, the larger the capacity and that the factors that most impact the capacity increase are the fraction of AVs in the traffic and the proportion of platooning-capable AVs in the AV fleet.

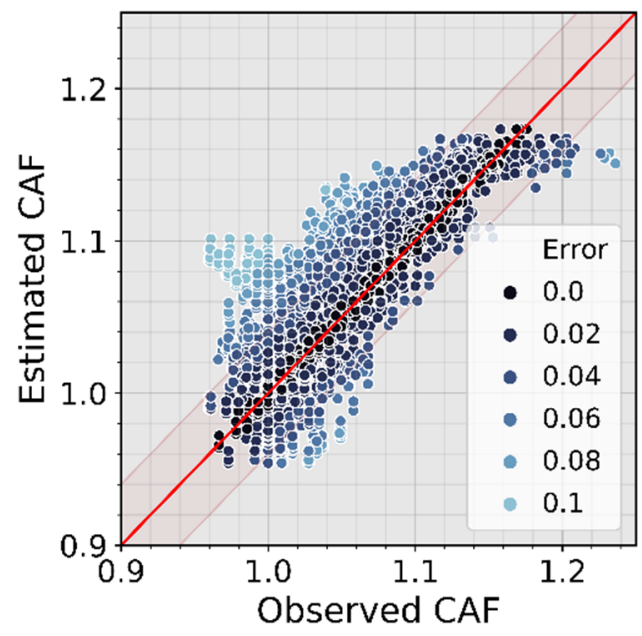

(a)

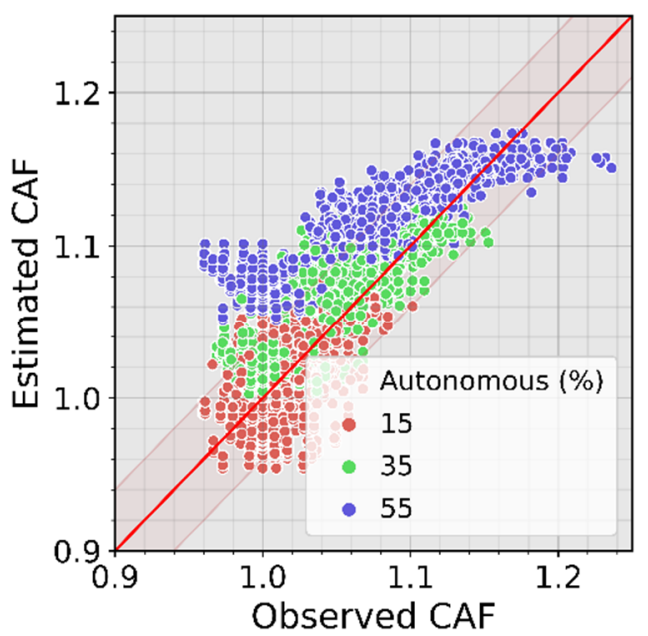

(c)

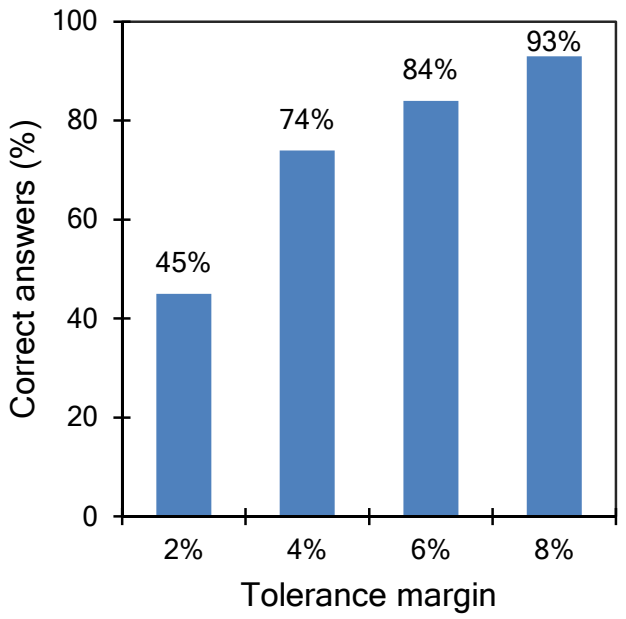

(b)

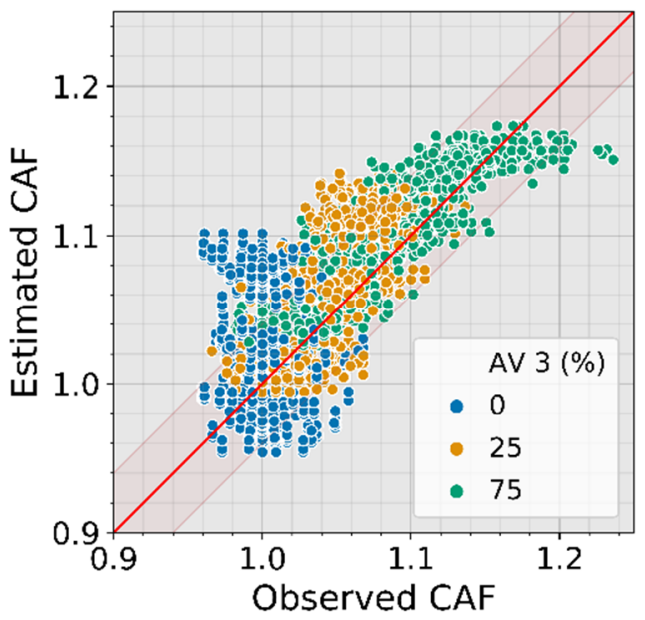

(d)

Figure 7. Validation of the model to estimate the CAF: (a) comparison between the estimated and the observed values of the dataset reserved for the model validation; (b) proportion of correct answers of the estimated CAF value; (c) comparison between estimated and observed values discretised by the proportion of AVs in the flow; and (d) comparison between estimated and discretised observed values by the proportion of type $3 \mathrm{AVs}$, in this case, an increase of $\mathrm{AV}_{3}$ implies a reduction in $\mathrm{AV}_{1}$.

The results of the interaction graphs suggest that the AVs platoons increase capacity and that the platoon maximum size does not affect the capacity for AV penetration levels up to $50 \%$. However, in scenarios with $60 \%$ of AVs in which platooning-capable AVs predominate, smaller platoons provide a significantly higher capacity than platoons with many AVs. For low levels of $\mathrm{AV}$ penetration (up to 30\%), the number of traffic lanes does not influence capacity; for AV fractions above $30 \%$, the capacity decreases as the number of traffic lanes increases. This occurs because a higher number of lanes leads to more lane changing manoeuvres (TRB, HCM p. 13-5), reducing the density and, consequently, the capacity. 
A CAF prediction model was adjusted using multivariate regression and its validation demonstrated a good performance of estimates, with mean errors of $3 \%$ to $4 \%$ of the $C A F$. Although a high rate of correct answers was obtained, the model may not be as precise for scenarios that are different from those simulated; therefore, it is suggested that future research uses simulations with different scenarios, different AV behaviours, inclusion of autonomous HGV and other characteristics of freeways.

The results indicate that AVs would increase the capacity of freeways in Brazil, and that the impact would be proportional to the fraction of AVs in the traffic. The research demonstrated that AVs with cooperative behaviour and greater V2V communication capabilities would lead to a greater increase in capacity, of up to $30 \%$ from the base capacity. These results are important for road system planning, freeway operation companies and future research in this area. However, it must be emphasized that these results are highly dependent on the microsimulation model adopted (Vissim); another model might produce different results.

\section{ACKNOWLEDGEMENTS}

The authors acknowledge the financial support from the Coordination for the Improvement of Higher Education Personnel Brazil (CAPES) - Financing Code 001 and from the National Council of Technological and Scientific Development (CNPq, Proc. 312460/17-1) for developing this research.

\section{REFERENCES}

Al-Kaisy, A. and C. Durbin (2011). Platooning on two-lane two-way highways: An empirical investigation, Procedia - Social and Behavioral Sciences, v. 16, p. 329-339. DOI:10.1016/j.sbspro.2011.04.454

Alkim, T. P., G. Bootsma and S. Hoogendoorn (2007). Field operational test - The assisted driver. Intelligent Vehicles Symposium, p. 1198-1203, Istambul: IEEE. DOI: 10.1109/IVS.2007.4290281

Bethônico, F. C., F. J. Piva and J. R. Setti (2016). Calibração de microssimuladores de tráfego através de medidas macroscópicas. Anais do XXX Congresso de Pesquisa e Ensino em Transportes. Rio de Janeiro: ANPET. Available at <http://146.164.5.73:30080/tempsite/anais/documentos/2018/Trafego Urbano e Rodoviario/Trafego em Rodovias II/4_487_AC.pdf> (accessed in 27/3/2021)

Bierstedt, J., A. Gooze, C. Gray, J. Peterman, L. Raykin and J. Walters (2014). Next-generation vehicles effects of on travel demand and highway capacity. Technical Report, Fehr and Peers, USA. Available at $<$ https://issuu.com/fehrandpeers/docs/fp_think_next_gen_vehicle_white_paper_final> (accessed in 27/3/2021)

Calvert, S. C., W. J. Schakel and J. W. van Lint (2017). Will automated vehicles negatively impact traffic flow? Journal of Advanced Transportation, v. 17, id 3082781, DOI:10.1155/2017/3082781.

Carvalho, L. G. S. and J. R. Setti (2019). Calibration of the Vissim truck performance model using GPS data. Transportes, v. 27, n. 3, p. 131-143, DOI:10.14295/transportes.v27i3.2042

Dowling, R., G. List, B. Yang, E. Witzke and A. Flannery (2014) Incorporating truck analysis into the Highway Capacity Manual, National Cooperative Freight Research Program (NCFRP) Report 31. Washington: TRB DOI:10.17226/22311

Fries, R., Y. Qi and S. Leight (2017) How many times should I run the model? Performance measure specific findings from VISSIM models in Missouri. In: 96th Annual Meeting of Transportation Research Board. Washington: TRB. Available at $<$ https://trid.trb.org/View/1437256> (accessed in 27/3/2021)

Gorter, M. (2015). Adaptive cruise control in practice a field study and questionnaire into its influence on driver, traffic flows and safety. Master Thesis, Delft. Available at <http://resolver.tudelft.nl/uuid:727070c8-c3c4-469f-a51a-a772ce683fa4> (accessed in 27/03/2021)

List, G. F., B. Yang and N. M. Rouphail (2015). On the treatment of trucks for analysis of freeway capacity. Transportation Research Record, v. 2483, p. 120-129. DOI:10.3141/2483-14

Mahmassani H. S. (2016) Autonomous vehicles and connected vehicle systems: flow and operations considerations. Transportation Science, v. 50, n. 4, p. 1140-1162, DOI: $10.1287 /$ trsc.2016.0712.

Makridis, M., K. Mattas, B. Ciuffo, M. A. Raposo, T. Toledo and C. Thiel (2018). Connected and automated vehicles on a freeway scenario. Effect on traffic congestion and network capacity. In 7th Transport Research Arena TRA, Vienna, 13 p. DOI: 10.5281/zenodo.1483132

Marcomini, L. A. and A. L. Cunha (2018). A comparison between background modelling methods for vehicle segmentation in highway traffic videos. arXiv:1810.02835 [cs.CV] - DOI: arXiv abs/1810.02835.

Martin-Gasulla, M., P. Sukennik and J. Lohmiller (2019). Investigation of the impact on throughput of connected autonomous vehicles with headway based on the leading vehicle type. Transportation Research Record, v. 2673, n. 5, p. 617-626. D0I: $0.1177 / 0361198119839989$

Milanés, V. and S. E. Shladover (2014). Modeling cooperative and autonomous adaptive cruise control dynamic responses using experimental data. Transportation Research Part C, v. 48, p. 285-300. DOI: 10.1016/j.trc.2014.09.001 
Papadoulis, A., M. Quddus, and M. Imprialou (2019). Evaluating the safety impact of connected and autonomous vehicles on motorways. Accident Analysis \& Prevention, v. 124, p. 12-22. DOI:10.1016/j.aap.2018.12.019

PTV (2019). Vissim 11 - User Manual. Technical Report, Karlsruhe.

Shi, Y., Q. He and Z. Huang (2019). Capacity analysis and cooperative lane changing for connected and automated vehicles: entropy-based assessment method. Transportation Research Record, v. 2673, n. 8, p. 485-498. DOI:10.1177/0361198119843474.

Shladover, S. E. (2009). Cooperative (rather than autonomous) vehicle-highway automation systems. IEEE Intelligent Transportation Systems Magazine, v. 1, n. 1, p. 10-19. DOI: 10.1109/MITS.2009.932716

Shladover, S. E., D. Su and X.-Y. Lu (2012). Impacts of cooperative adaptive cruise control on freeway traffic flow. Transportation Research Record, v. 2324, n. 1, p. 63-70. DOI: 10.3141/2324-08

Strand, N, J. Nilsson, I. Karlsson and L. Nilsson (2011). Exploring end-user experiences: self-perceived notions on use of adaptive cruise control systems. IET Intelligent Transport Systems, v. 5, n. 2, p. 134-140. DOI: 10.1049/iet-its.2010.0116

Sukennik, P. (2018). Micro-simulation guide for automated vehicles. PTV - CoExist, Technical Report, available at <https://www.h2020-coexist.eu/wp-content/uploads/2018/11/ D2.5-Micro-simulation-guide-for-automated-vehicles.pdf> (accessed in 27/3/2021)

TRB (2016). Highway Capacity Manual (6 $6^{\text {th }}$ ed.). Washington, D.C.: TRB.

Van Arem, B., C. J. G. Van Driel and R. Visser (2006). The impact of cooperative adaptive cruise control on traffic-flow characteristics. IEEE Transactions on Intelligent Transportation Systems, v. 7, n. 4, p. 429-436. DOI: 10.1109/TITS.2006.884615

Vanderwerf, J., S. Shladover and M. A. Miller (2004). Conceptual development and performance assessment for the deployment staging of advanced vehicle control and safety systems. Technical Report. Berkeley: University of California. Available at $<$ https://escholarship.org/content/qt8hg3b55r/qt8hg3b55r.pdf $\geq$ (visited on 15/3/2021)

Xiao-bao, Y. and Z. Ning (2007). Effects of the Number of Lanes on Highway Capacity, International Conference on Management Science and Engineering, p. 351-356, Harbin: IEEE. DOI: 10.1109/ICMSE.2007.4421872

Zhao, L. and J. Sun (2013). Simulation framework for vehicle platooning and car-following behaviours under connected-vehicle environment. Procedia - Social and Behavioral Sciences, v. 96, p. 914-924. DOI: 10.1016/j.sbspro.2013.08.105

Zhou, J., L. Rilett and E. Jones (2019a). Sensitivity analysis of speed limit, truck lane restrictions, and data aggregation level on the HCM-6 passenger-car equivalent estimation methodology for western U.S. conditions. Transportation Research Record, v. 2673, n. 11, p. 493-504. DOI: 10.1177/0361198119851451.

Zhou, J., L. Rilett, and E. Jones (2019b). Estimating passenger car equivalent using the HCM-6 PCE methodology on four-lane level freeway segments in western U.S. Transportation Research Record, v. 2673, n. 11, p. 529-545. DOI: $10.1177 / 0361198119851448$

Alessandri, A., A. Di Febbraro, A. Ferrara and E. Punta (1998) Optimal Control of Freeways via Speed Signalling and Ramp Metering. Control Engineering Practice, v. 6, n. 6, p. 771-780. DOI: 10.1016/S0967-0661(98)00083-5. 